\title{
Synthesis and Characterization of Nanoparticles and Thin Films of PbS by a High-performance Procedure Using CBD
}

Beatriz Guadalupe Zaragoza-Palacios ( $\sim$ beatriz.zaragoza@fisica.uson.mx )

Universidad de Sonora https://orcid.org/0000-0003-0196-4031

Angel Roberto Torres-Duarte

Universidad de Sonora

Santos Jesús Castillo

Universidad de Sonora

\section{Research Article}

Keywords: Lead sulfide, nanoparticles, thin films, thioacetamide, ammonia-free, semiconductors

Posted Date: March 4th, 2021

DOI: https://doi.org/10.21203/rs.3.rs-271679/v1

License: (c) (i) This work is licensed under a Creative Commons Attribution 4.0 International License.

Read Full License

Version of Record: A version of this preprint was published at Journal of Materials Science: Materials in Electronics on August 4th, 2021. See the published version at https://doi.org/10.1007/s10854-02106702-y. 


\section{Synthesis and characterization of nanoparticles and thin films of PbS by a high-performance procedure using CBD}

Beatriz Guadalupe Zaragoza-Palacios ${ }^{\mathrm{a}}$, Angel Roberto Torres-Duarte ${ }^{\mathrm{b}}$ and Santos Jesús Castillob

a Departamento de Física, Universidad de Sonora, Hermosillo, Sonora, México, C.P. 83000.

b Departamento de Investigación en Física, Universidad de Sonora, Hermosillo, México, C.P. 83000.

Corresponding author:

Beatriz Guadalupe Zaragoza-Palacios

ORCID: 0000-0003-0196-4031

E-mail: beatriz.zaragoza@unison.mx

Departamento de Física

Universidad de Sonora

Blvd. Luis Encinas y Blvd. Rosales S/N

Apartado Postal 1626

Hermosillo, Sonora, México

C. P. 83000

\section{Highlights}

- The synthesis of PbS nanoparticles and thin film employed lead acetate as lead source and thioacetamide as sulfide source, and polyethyleneimine as the complexing agent. 
- PbS nanoparticles synthesized at room conditions were made in a few minutes.

- Homogeneous nanoparticles less than $10 \mathrm{~nm}$ and a bandgap of $3.35 \mathrm{eV}$ were obtained.

- PbS nanostructured thin films were elaborated by chemical bath deposition at $50^{\circ} \mathrm{C}$ in one hour.

- A homogeneous thin film around $160 \mathrm{~nm}$ of thickness is formed of small PbS crystallites of $22 \mathrm{~nm}$ approximately and bandgap of $1.85 \mathrm{eV}$ was obtained.

- Sodium hydroxide was used to maintain the $\mathrm{pH}$, instead of ammonium or ammonia hydroxide to make thin films of $\mathrm{PbS}$.

\section{Abstract.}

In this research, nanoparticles and nanostructured thin films of lead sulfide are synthesized by using physicochemical methods at room conditions or suchlike conditions, at short reaction times; furthermore, is used simple laboratory equipment and a simplified experimental configuration. Both syntheses do not employ, ammonium or ammonia to elaborate $\mathrm{PbS}$. The main used precursors for both processes are lead acetate, as the lead source, polyethyleneimine as the complexing agent, and thioacetamide, as the sulfur source. Acetylacetone has been employed as a dispersant agent to elaborate PbS nanoparticles. While the sodium hydroxide maintains a high $\mathrm{pH}$, to elaborate $\mathrm{PbS}$ thin films. The characterizations reveal the existence of $\mathrm{PbS}$ nanoparticles whose size is smaller than $10 \mathrm{~nm}$ and with 
a band gap of $3.35 \mathrm{eV}$. On the other hand, the resulting thin film is made up of $\mathrm{PbS}$ little crystallites of $22 \mathrm{~nm}$ in a homogeneous way and its band gap is $1.85 \mathrm{eV}$.

\section{Keywords}

Lead sulfide; nanoparticles; thin films; thioacetamide; ammonia-free; semiconductors.

\section{Introduction}

The semiconductors are materials used in different electronic devices, according to their electronic properties, like bandgap or conductivity, for example. Particularly, the $\mathrm{PbS}$ is a chalcogenide material that can change these properties by reducing its size to nanometric scales. Specifically, it is possible to change its bandgap from the infrared region to the ultraviolet region, in contrast, the bulk value is $0.37 \mathrm{eV}$. Consequently, the PbS can be employed in devices like solar cells, LEDs, photocatalysis, photoluminescence, or infrared photodetectors. Despite, many kinds of research that have been performed, from their synthesis processes, their characterizations, until the applications, however, it is still competing to find an effective and low-cost synthesis.

There are reported applications for PbS on photocell as patent since 1948 by R. J. Cashman [1]. Thus, solar cells with different structures had been elaborated [2] [3]. There are works where detectors are produced with $\mathrm{PbS}$ colloidal quantum dot photoconductors [4]. In 2017 Priyanka realized the synthesis biologically of PbS 
nanoparticles and applied it to detect arsenic(III) in water, see [5]. Also, PbS quantum dots have been used as contrast materials in laboratory studies in tumors, checking their low cytotoxicity, there are researches related to this [6]. Moreover, there are different methods of synthesis, for example, Khan et al. reported the deposition of $\mathrm{PbS}$ thin films via aerosol assisted chemical vapor deposition [7]. In recent times, mechanochemical synthesis of PbS nanocrystals from lead oxide [8] and, a chemical synthesis for PbS nanoparticles [9] were reported.

This research is focused on developing chemical processes for growing $\mathrm{PbS}$ nanoparticles, as well as PbS nanostructured thin films, and realize their fundamental characterization to verify the identity of the obtained compounds and their optical properties. The work proposes a first-principles technique to elaborate $\mathrm{PbS}$ nanoparticles and PbS nanostructured thin films, with room conditions or close to them, a few reagents, ammonia, and ammonium free, short reaction times, and being affordable.

\section{Material and methods}

In this article, a synthesis method to elaborate both nanoparticles and thin films of $\mathrm{PbS}$ is presented. This method was realized at room temperature or close to it, in short times. This method mainly consists of an aqueous solution containing a diluent, a complexing agent, a source of lead, and a source of sulfur, for both materials. For this method, different agents can be used but, in this work, particularly those in table 1 were used.

Table 1. Reagents employed to both synthesis, $\mathrm{PbS}$ nanoparticles and $\mathrm{PbS}$ thin film. 


\begin{tabular}{ccc}
\hline Reagents & Molar concentration & Volume \\
\hline Deionized water & $\bullet$ & $\bullet$ \\
Thioacetamide & $\bullet$ & $\cdot$ \\
Lead acetate & $\bullet$ & $\bullet$ \\
Polyethyleneimine & $\bullet$ & - \\
Acetylacetone & $\bullet$ & $\cdot$ \\
Sodium hydroxide & - & \\
\hline
\end{tabular}

From Table 1, it can be seen that using essentially the same reagents, it is possible to obtain two forms for PbS: nanoparticles and thin films. Hence, if we want to synthesize PbS nanoparticles, we add acetylacetone to the aqueous solution, as a diluent. On the other hand, if we want to make PbS thin films, we add sodium hydroxide to the chemical bath to maintain the $\mathrm{pH}$. The equipment used for the nanoparticles is only a test tube and ultrasonic bath equipment, while for the thin films Corning glass substrates, a heating plate, and a bath container were used. (Scheme 1).

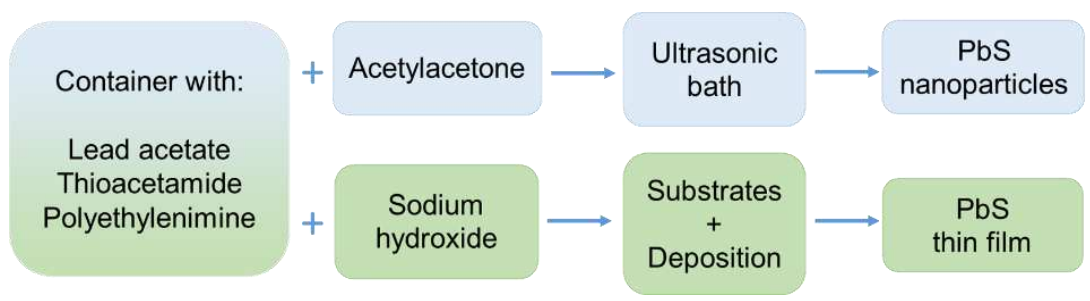

Scheme 1. Synthesis diagram to make PbS nanoparticles or PbS thin films.

Continuing, are explained the employed formulations for each system, the used precursors, and the used equipment for the characterization.

\subsection{PbS nanoparticles}


The used reactor was a $30 \mathrm{ml}$ test tube, the first reagent employed was $100 \mathrm{ml}$ of acetylacetone $(0.025 \mathrm{M})$, next $1 \mathrm{ml}$ of lead acetate $(0.5 \mathrm{M})$ were added, then $1.5 \mathrm{ml}$ of polyethyleneimine $(x M)$, and finally $1 \mathrm{ml}$ of thioacetamide $(0.1 \mathrm{M})$. Once added reagents, the test tube was collocated into an ultrasonic bath for 20 minutes, the reaction temperature was of the laboratory $\left(24^{\circ} \mathrm{C}\right)$, after that, some color changes were observed until they were stable around 30 minutes. The used polyethyleneimine is a kind of branched compound, that makes it difficult to calculate its molarity, but we dissolve 3.5 milliliters of the pure polyethyleneimine compound in $50 \mathrm{ml}$ of deionized water and this is denominated " $x \mathrm{M}$ ". Acetylacetone has a double purpose, is a complexing agent and a dispersant; the lead acetate is the lead source, the polyethyleneimine is the complexing agent, and the sulfur source is the thioacetamide.

\subsection{PbS thin films}

The PbS thin films were synthesized by chemical bath deposition on a soda-lime glass substrate, corning brand. The used reactor was a $100 \mathrm{ml}$ beaker, first $5 \mathrm{ml}$ of lead acetate $(0.5 \mathrm{M})$ was poured, second $5 \mathrm{ml}$ of polyethyleneimine $(x \mathrm{M})$, then $1 \mathrm{ml}$ sodium hydroxide $(2 \mathrm{M})$, next $5 \mathrm{ml}$ thioacetamide $(0.1 \mathrm{M})$ and finally $40 \mathrm{ml}$ deionized water. In this system, sodium hydroxide has the purpose to increase the $\mathrm{pH}$, while the other reagents have the same functionality as for nanoparticles system. The deposition temperature was $50^{\circ} \mathrm{C}$. The glass substrates were located in a vertical position for 60 minutes. The deposited $\mathrm{PbS}$ thin film has a dark color, almost black, and presents a well adhered to substrate and reflective behavior.

\subsection{Equipment}


The method of synthesis for both $\mathrm{PbS}$ nanostructures employed essentially a container with the reagents. Additionally, the reaction of PbS nanoparticles was performed inside an ultrasonic bath, while the $\mathrm{PbS}$ thin film was elaborated in a chemical bath deposition.

The equipment used for characterizations was JEOL TEM model JEM 2010F, VWR spectrophotometer model UV-6300PC, JEOL SEM model JSM-7800F and Raman spectrophotometer Horiba model LabRam HR.

\section{Results}

\subsection{PbS nanoparticles}

This section begins with characterizations of nanoparticles leading to the formal identification of the synthesized chemical compound, Figure 1 displays a set of micrograph images coming from transmission electron microscopy, the images 1a1c correspond to three different work scale as is indicated, a kind of branched behavior adopted for the small cluster is observed at this sequence. In Figure 1c, the size of the particles is almost homogeneous. 

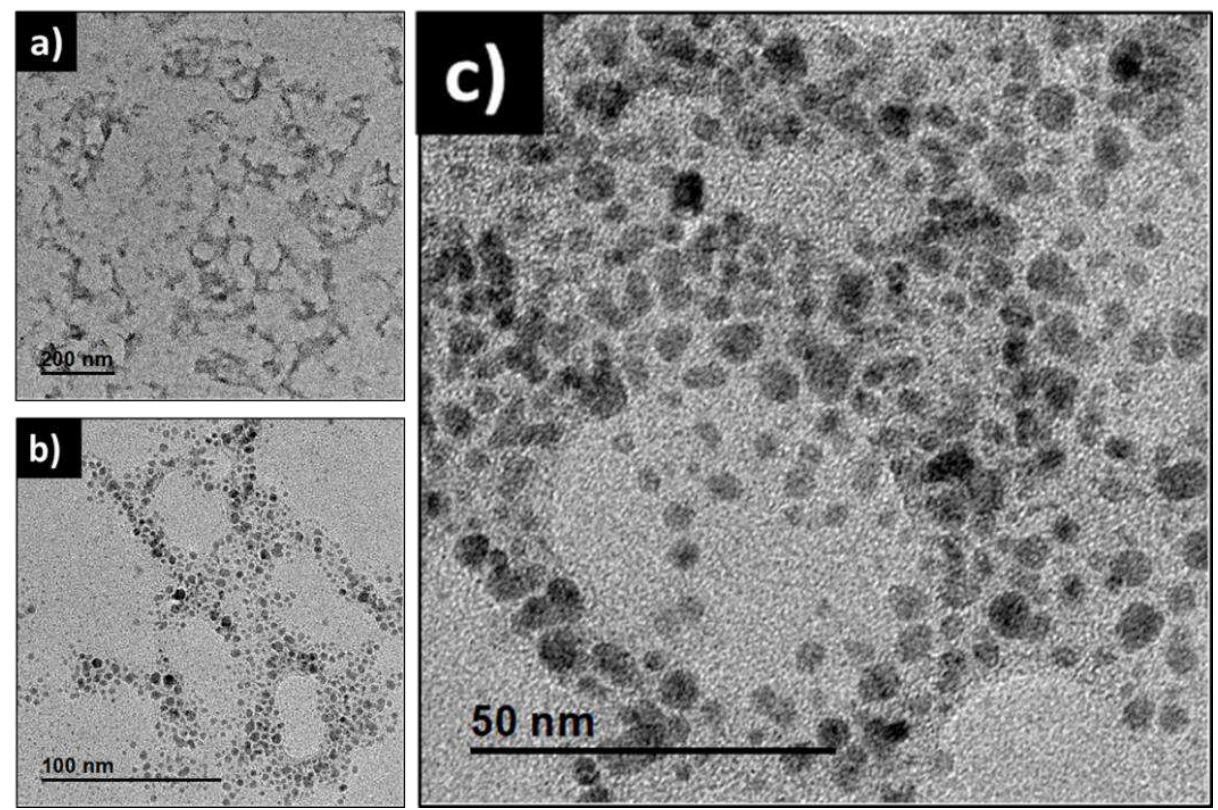

Fig. 1. Transmission electron micrographics of PbS nanoparticles at different work scales (1a-1c), as indicated.

With HRTEM it is possible to appreciate both the size of the nanoparticles and the interplanar distance. Fig. 2a shows nanoparticles are smaller than $5 \mathrm{~nm}$. The contrast micrograph (Fig. 2b) exhibits the crystalline planes in the small cluster of three nanoparticles. Finally, in Fig. $2 \mathrm{c}$ is plotted the Fast Fourier transform (FFT) of $2 \mathrm{~b}$, where reciprocal distances between dot pairs are indicated.
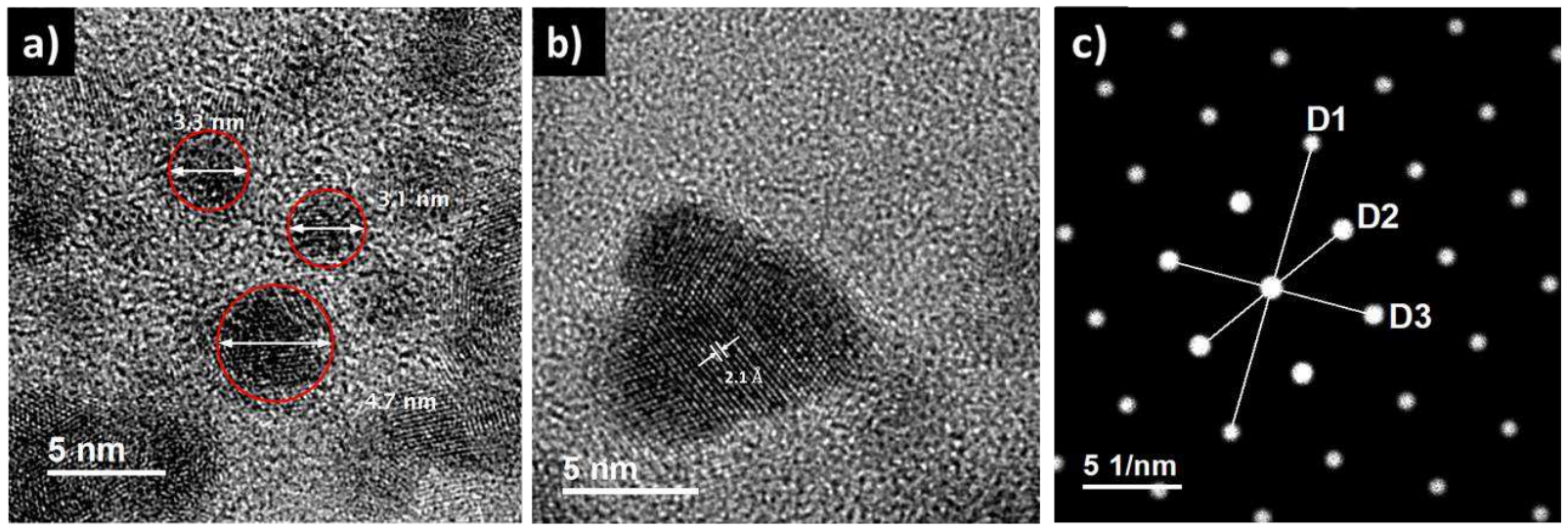
Fig. 2. a) HRTEM micrograph with some nanoparticles, b) HRTEM micrograph shows the interplanar distance of nanoparticles and, c) Reciprocal space of b)

The results are indicated in table 2, first column data correspond to reciprocal distances of dot pairs at the Fourier space of the Fast Fourier Transform obtained from the contrast real image of Figure 2c. The second column is calculated from the first column and shows three different interplanar distances matching with database PDF 77-0244 and corresponding Miller indexes (column 3) for lead sulfide.

Table 2. Reciprocal length and interplanar distance to micrograph HRTEM of PbS nanoparticles and the corresponding Miller indexes.

\begin{tabular}{ccc}
\hline $\begin{array}{c}\text { Reciprocal length } \\
(\mathbf{1} / \mathbf{n m})\end{array}$ & $\begin{array}{c}\text { Calculated interplanar } \\
\text { distance }(\AA)\end{array}$ & PDF 77-0244 \\
\hline D1 $=15.138$ & 1.32 & 420 \\
D2 $=9.400$ & 2.13 & 220 \\
D3 $=10.799$ & 1.85 & 311 \\
\hline
\end{tabular}

Figure 3 depicts the behavior of two fundamental optical responses, the absorption (in arbitrary units) and the transmission (in percentage proportions), the plot a) illustrates their correlation with wavelength, while the plot b) their dependence with the involved energy. As can be observed there exists an absorption edge in the visible region, which can be due to the nanometric nature of the particles. 

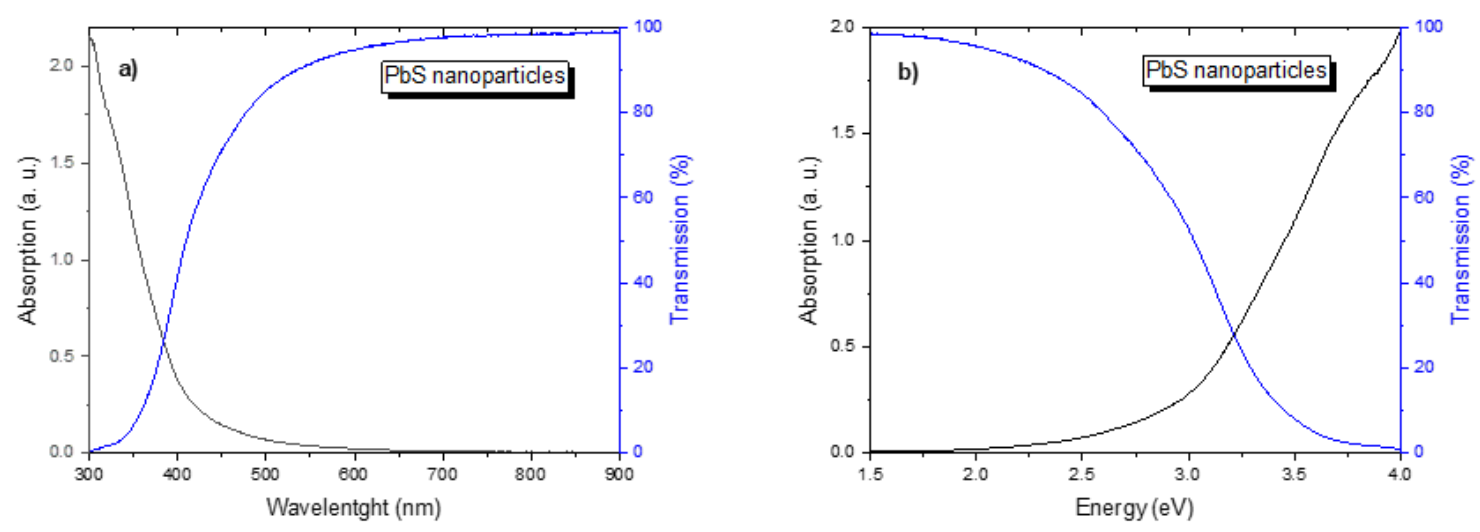

Fig. 3. The behavior of absorption and transmission of the PbS nanoparticles, part a) depending on wavelength and part b) as a function of energy.

From the data absorption is possible to apply the well-known Tauc process [10] [11] [12] to obtain the energy band gap value, Figure 3 illustrates the direct bandgap estimated of $3.35 \mathrm{eV}$ with a good approach correlation coefficient, and located at the intersection of the linear fitting, with the energies axis. 


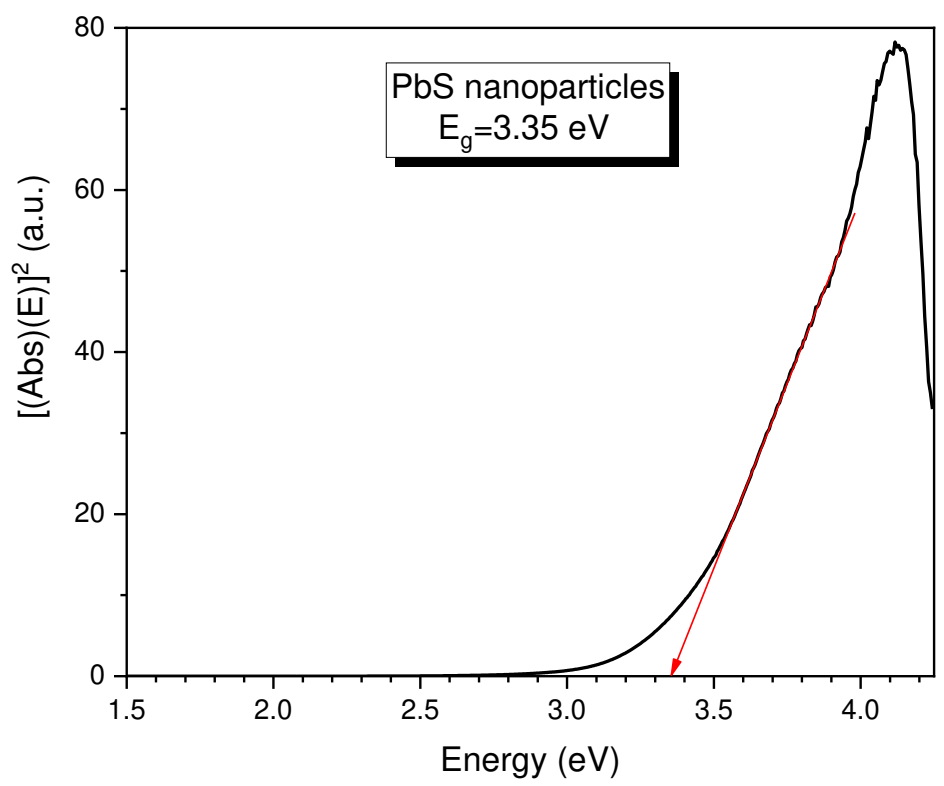

Fig. 4. Graphical support of the direct bandgap calculation of PbS nanoparticles in the visible region, using the Tauc method.

\subsection{PbS thin films.}

The PbS thin film characterizations include SEM images, XRD pattern, the optical responses of UV-vis, and Raman spectroscopy. Figure 5 depicts three SEM images, the a) part is a top view of the surface morphology for a PbS thin film obtained through the process with a work scale of $1 \mu \mathrm{m}$ as a result of magnification of $x 10,000$. The b) and c) part correspond to work scale of $100 \mathrm{~nm}$ but b) part magnification is $x 50,000$ while the c) part magnification is $x 100,000$ and here is possible to observe various isolated cluster cubic shaped $\mathrm{PbS}$ which form a characteristic roughness on the surface. As can be observed, the surface morphology is homogeneous and 
formed of small PbS crystallites. There exists in the scientific literature, reported different morphologies for PbS thin film, see [13] [14] [15].
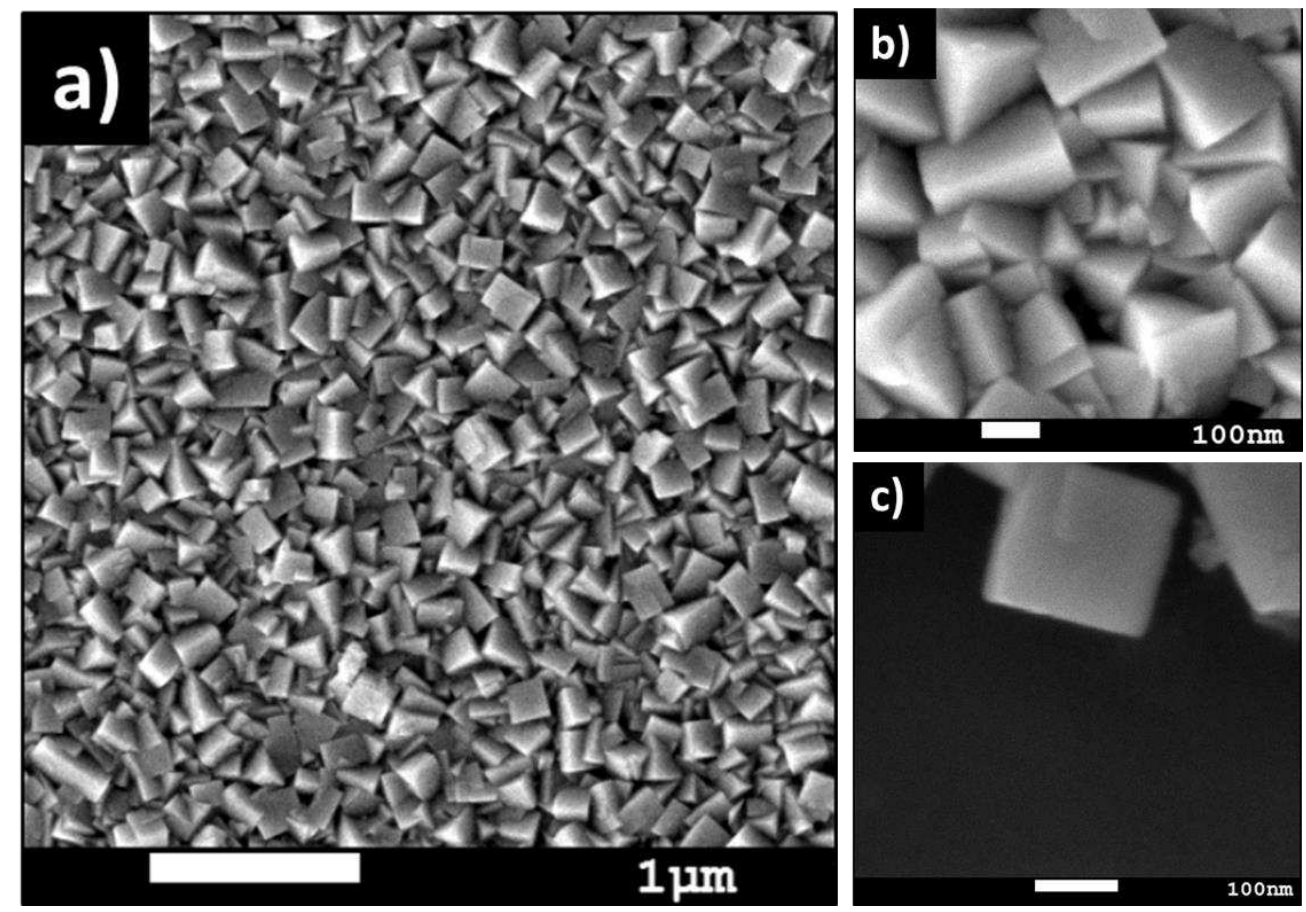

Fig. 5. Top view of surface for PbS thin film to different magnifications, a) 10,000x, b) $50,000 x$ and c) $100,000 x$.

Figure 6 presents the SEM image in a cross-section view, where it can be observed the thickness of the PbS thin film grown of $160 \mathrm{~nm}$, the down part of the image is the substrate. 


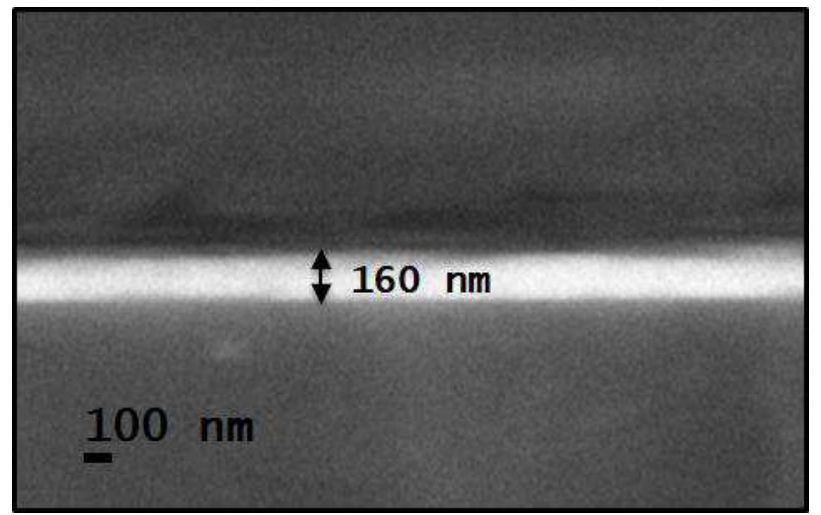

Fig. 6. SEM image of cross-section views, estimating the thickness of PbS thin film.

Furthermore, Figure 7 gives the elemental distribution of lead and sulfide in the $\mathrm{PbS}$ thin film that shows a clear presence of each one, taken by an EDS elemental mapping equipment.

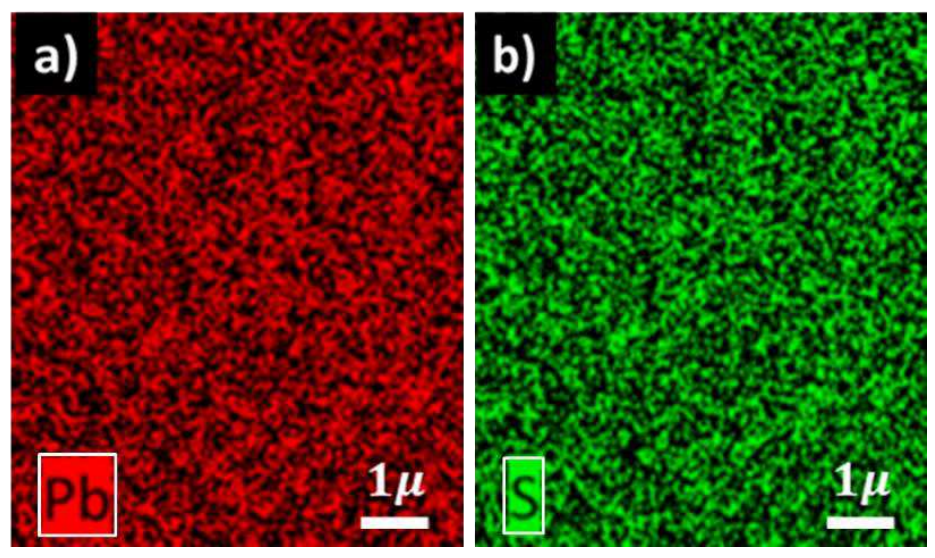

Fig. 7. Elemental distribution on PbS thin film, a) lead and b) sulfide.

The observed transmission and absorption responses are located in the visible region, where the seen behavior presents a bandgap transition. Figure 8 a) is shown 
those responses, while Fig. 8 b) depicts the graphical, lie on analytical calculations, the direct bandgap value of our PbS thin film in the indicated region $E_{g}=1.85 \mathrm{eV}$. This optical band gap can be explained due to the nanostructured nature of the thin film.
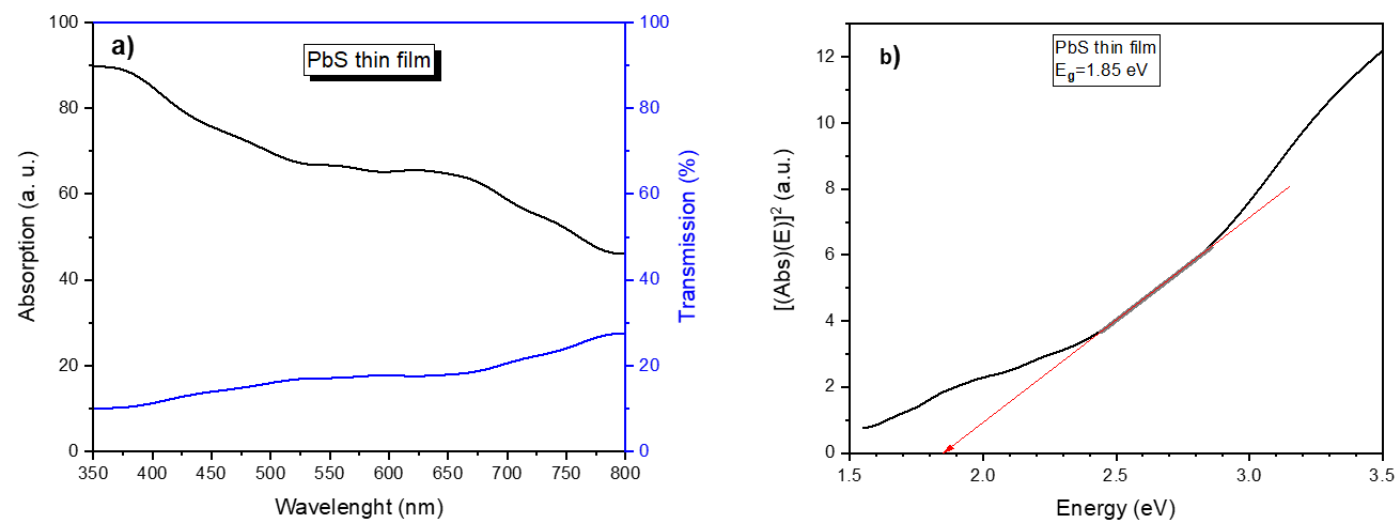

Fig. 8. Part a) comparative complementary behaviors of absorption and transmission for a PbS thin film, and part b) graphical support for direct bandgap calculation.

Figure 9 depicts the XRD pattern collected with razing incidence for PbS thin film, as can be observed, there is a good definition for the peaks at $2 \theta=30.36^{\circ}, 35.16^{\circ}$, $50.56^{\circ}, 60.08^{\circ}$, and $63.16^{\circ}$; those peaks correspond to crystallographic planes (11 1), (2 0 0), (2 2 0), (3 11 1) and (2 2 2), respectively. This pattern was collected using an X-ray cobalt source, matching with PDF 78-1897. From the maximum intensity peak, it is plausible to calculate the size of the crystallite corresponding to this pattern by using the Debye-Scherrer equation [16].

$$
\text { Crystallite size }=\frac{0.94 \lambda}{\beta(\mathrm{rad}) \operatorname{Cos} \theta}
$$


Here $\lambda=1.54 \AA ; \beta=0.0064 \mathrm{rad} ; \theta=15.185^{\circ}$.

Substituting these values at the Debye-Scherrer equation we obtained the crystallite size of $22 \mathrm{~nm}$, consequently, the small cubic formations observed in Figures $5 \mathrm{~b}$ ) and 5c) should be PbS clusters.

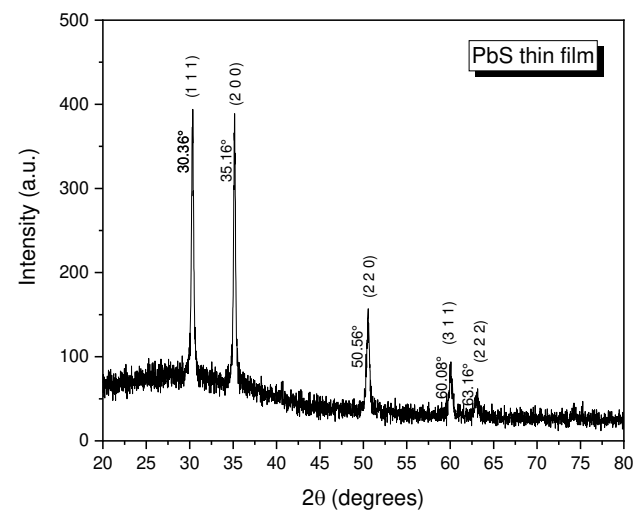

Fig. 9. XRD pattern of polycrystalline PbS thin film deposited on a soda-lime glass substrate.

From Raman dispersion spectroscopy, the signals shown in Figure 10 were obtained, this shape corresponds with those reported by [17] for PbS thin film. The band around $200 \mathrm{~cm}^{-1}$ and $430 \mathrm{~cm}^{-1}$ matches the $\mathrm{PbS}$ compound, while the high peak in $140 \mathrm{~cm}^{-1}$ is proof of the presence of PbS nanocrystallites. Therefore, this supports identifying the films formed by the binary lead sulfide compound. 


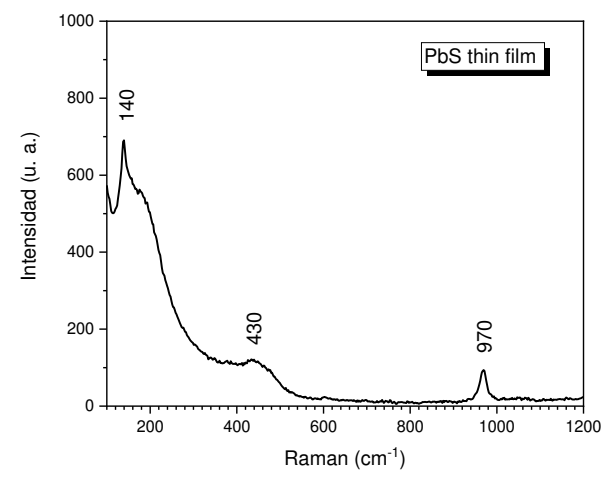

Fig. 10. Raman dispersion spectroscopy was collected from synthesized PbS thin film, using a $488 \mathrm{~nm}$ laser, with three characteristic values corresponding to the $\mathrm{PbS}$ compound.

\section{Conclusion.}

In this article, we have presented a successful process to elaborate $\mathrm{PbS}$ nanoparticles with an aqueous solution reaction at room conditions in 10 minutes. In this reaction, the main precursors are lead acetate as the lead source, thioacetamide as the sulfur source, and polyethyleneimine as the complexing agent. The TEM micrographs images showed the presence of small clusters, while the HRTEM images showed PbS nanoparticles smaller than $10 \mathrm{~nm}$. The obtained Fast Fourier Transform identified the crystallographic planes corresponding to the database PDF 77-0244. These nanoparticles were also characterized by a UV-vis spectrometer to obtain the corresponding absorption and transmission curves. From these results and using the Tauc method, the analysis was made to calculate the energy gap, obtaining a result of $\mathrm{Eg}=3.35 \mathrm{eV}$. 
While for PbS thin films, a successful formulation film by chemical bath reactions at $50^{\circ} \mathrm{C}$ in 1 hour was obtained, using also lead acetate, thioacetamide, and polyethyleneimine. The SEM images showed a homogeneous surface morphology formed of small PbS crystallites of thickness approximately $160 \mathrm{~nm}$. The XRD pattern corresponds to PDF 78-1897 and the Debye-Scherrer throws a crystallite size of $22 \mathrm{~nm}$. The bandgap obtained from spectroscopy UV-vis is $1.85 \mathrm{eV}$. Finally, Raman spectroscopy confirmed the presence of the binary lead sulfide compound.

\section{Declarations.}

The authors received no financial support for the research, authorship, and/or publication of this article.

The authors declare that they have no known competing financial interests or personal relationships that could have appeared to influence the work reported in this paper.

The data that support the findings of this study are available from the corresponding author, B. G. Zaragoza-Palacios, on request.

\section{Acknowledgments.}

We gratefully acknowledge the use of TEM facilities at the TEM Laboratory of Universidad de Sonora, as well as to the Laboratorio de Bionanoingeniería for allowing the use of VWR Spectrophotometer UV-6300PC UV-vis double beam, also we appreciate the Posgrado en Nanotecnologia of Universidad de Sonora for the facilities for the use of spectrometry equipment. 
Also, Beatriz G. Zaragoza Palacios acknowledges CONACyT for the scholarship $863944 / 637634$.

\section{References}

[1] R. J. Cashman, "Photocell of lead sulfide", 2,448,516, Sept; 7, (1948) United States Patent Office

[2] F. Göde and S. Ünlü, Mater. Sci. Semicond. Process., vol. 90, no. September 2018, pp. 92-100 (2019) doi: 10.1016/j.mssp.2018.10.011.

[3] X. Yang et al., Nano-Micro Lett., vol. 9, no. 2 (2017) doi: 10.1007/s40820016-0124-2.

[4] A. De lacovo, C. Venettacci, L. Colace, L. Scopa, and S. Foglia, IEEE Photonics Technol. Lett., vol. 29, no. 9, pp. 703-706 (2017) doi: 10.1109/LPT.2017.2680741.

[5] U. Priyanka, A. Gowda K M, E. M G, S. Teja B, N. Nitish, and R. Mohan B, Int. Biodeterior. Biodegrad., vol. 119, pp. 78-86 (2017) doi: 10.1016/j.ibiod.2016.10.009.

[6] J. Cao et al., J. Biomed. Mater. Res. - Part A, vol. 100 A, no. 4, pp. 958-968 (2012) doi: 10.1002/jbm.a.34043.

[7] M. D. Khan et al., Mater. Sci. Semicond. Process., vol. 46, pp. 39-45 (2016) doi: 10.1016/j.mssp.2016.02.002.

[8] W. Meng et al., Powder Technol., vol. 347, pp. 130-135 (2019) doi: 10.1016/j.powtec.2019.02.035.

[9] C. K. Miskin et al., ACS Applied Nano Materials, 2 (3), 1242-1252 (2019) doi: 10.1021/acsanm.8b02125.

[10] J. D. Patel, F. Mighri, and A. Ajji, Mater. Res. Bull., vol. 47, no. 8, pp. 20162021 (2012) doi: 10.1016/j.materresbull.2012.04.007.

[11] D. C. Onwudiwe, Heliyon, vol. 5, no. 3, p. e01413 (2019) doi: 10.1016/j.heliyon.2019.e01413.

[12] K. P. Mubiayi, N. Revaprasadu, S. S. Garje, and M. J. Moloto, J. Saudi Chem. Soc., vol. 21, no. 5, pp. 593-598 (2017) doi: 10.1016/j.jscs.2017.02.002.

[13] F. Göde, F. Yavuz, and I. A. Kariperb, Acta Phys. Pol. A, vol. 128, no. 2, pp. 215-218 (2015) doi: 10.12693/APhysPolA.128.B-215.

[14] S. Rajathi, K. Kirubavathi, and K. Selvaraju, Arab. J. Chem., vol. 10, no. 8, pp. 1167-1174 (2017) doi: 10.1016/j.arabjc.2014.11.057. 
[15] A. N. Fouda, M. Marzook, H. M. Abd El-Khalek, S. Ahmed, E. A. Eid, and A. B. El Basaty, Silicon, vol. 9, no. 6, pp. 809-816 (2017) doi: 10.1007/s12633015-9399-z.

[16] R. Bai, D. Kumar, S. Chaudhary, and D. K. Pandya, Acta Mater., vol. 131, pp. 11-21 (2017) doi: 10.1016/j.actamat.2017.03.062.

[17] A. Slonopas, N. Alijabbari, C. Saltonstall, T. Globus, and P. Norris, Electrochim. Acta, vol. 151, pp. 140-149 (2015) doi:

10.1016/j.electacta.2014.11.021. 


\section{Figures}

\section{Figure 1}
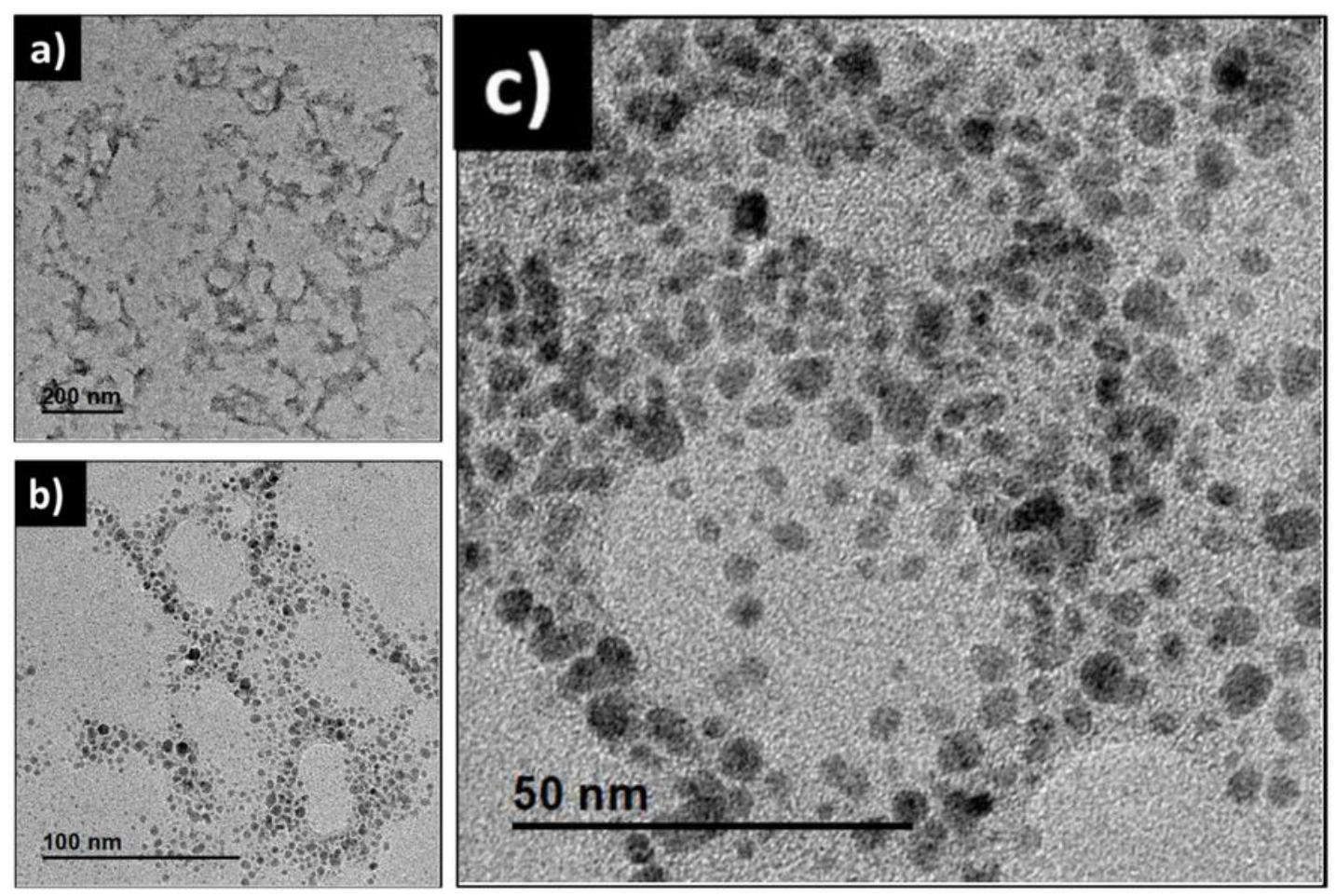

\section{Figure 1}

Transmission electron micrographics of PbS nanoparticles at different work scales (1a-1c), as indicated. 


\section{Figure 2}
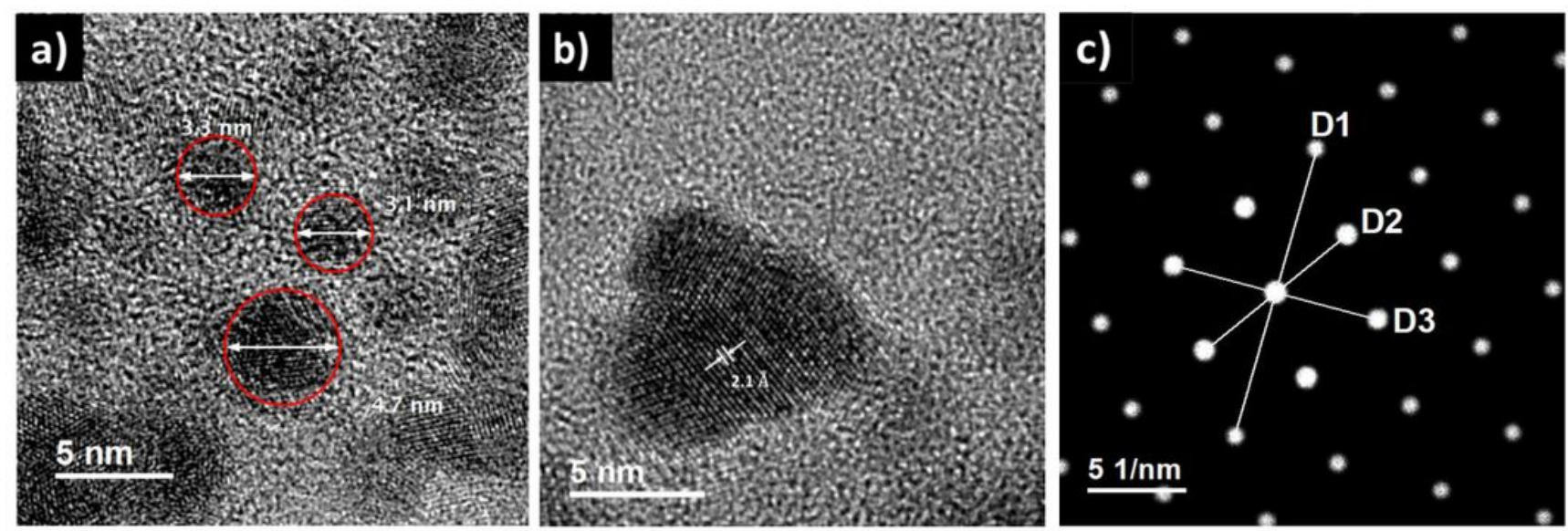

Figure 2

a) HRTEM micrograph with some nanoparticles, b) HRTEM micrograph shows the interplanar distance of nanoparticles and, c) Reciprocal space of b) 


\section{Figure 3}
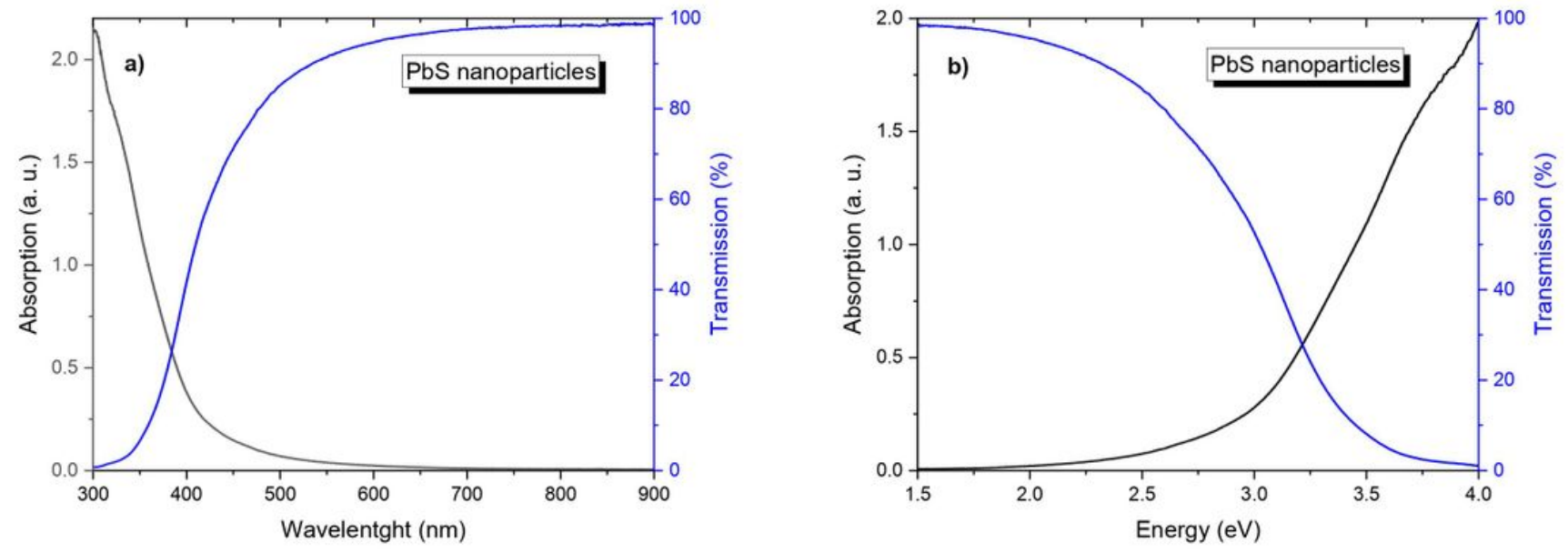

\section{Figure 3}

The behavior of absorption and transmission of the PbS nanoparticles, part a) depending on wavelength and part b) as a function of energy. 


\section{Figure 4}

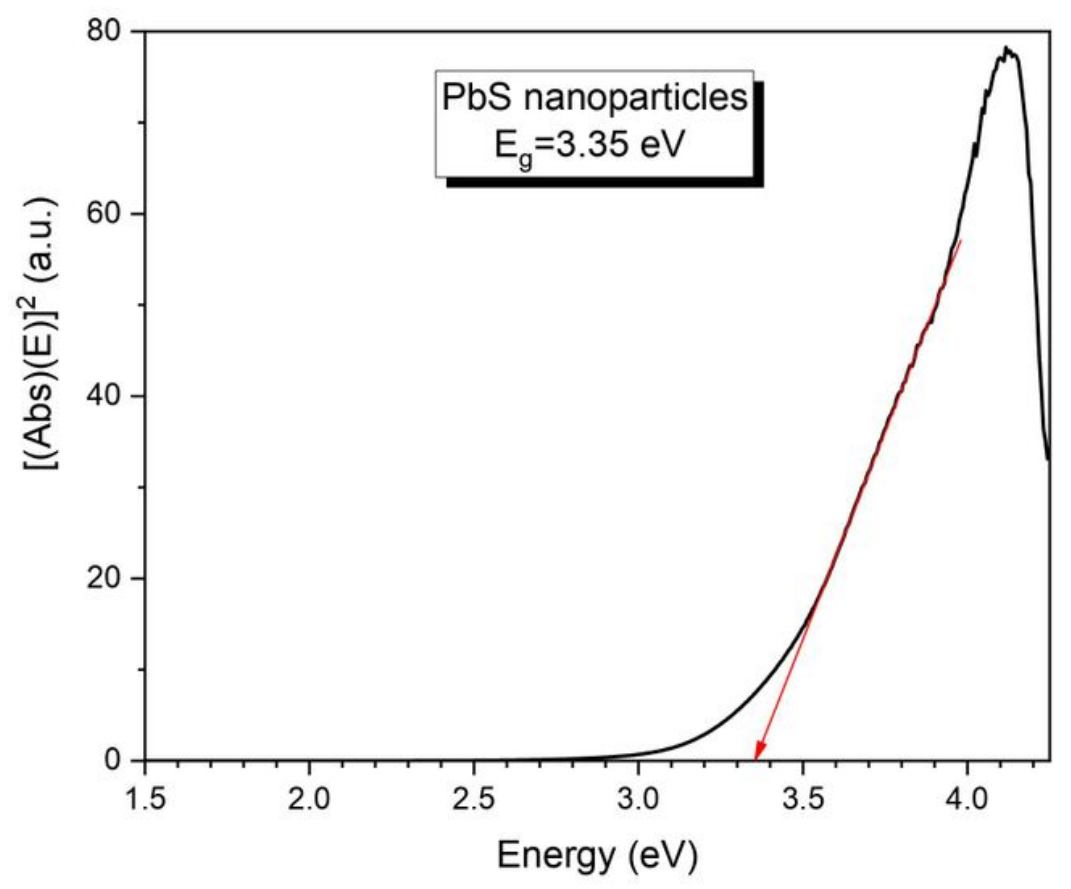

Figure 4

Graphical support of the direct bandgap calculation of PbS nanoparticles in the visible region, using the Tauc method. 


\section{Figure 5}
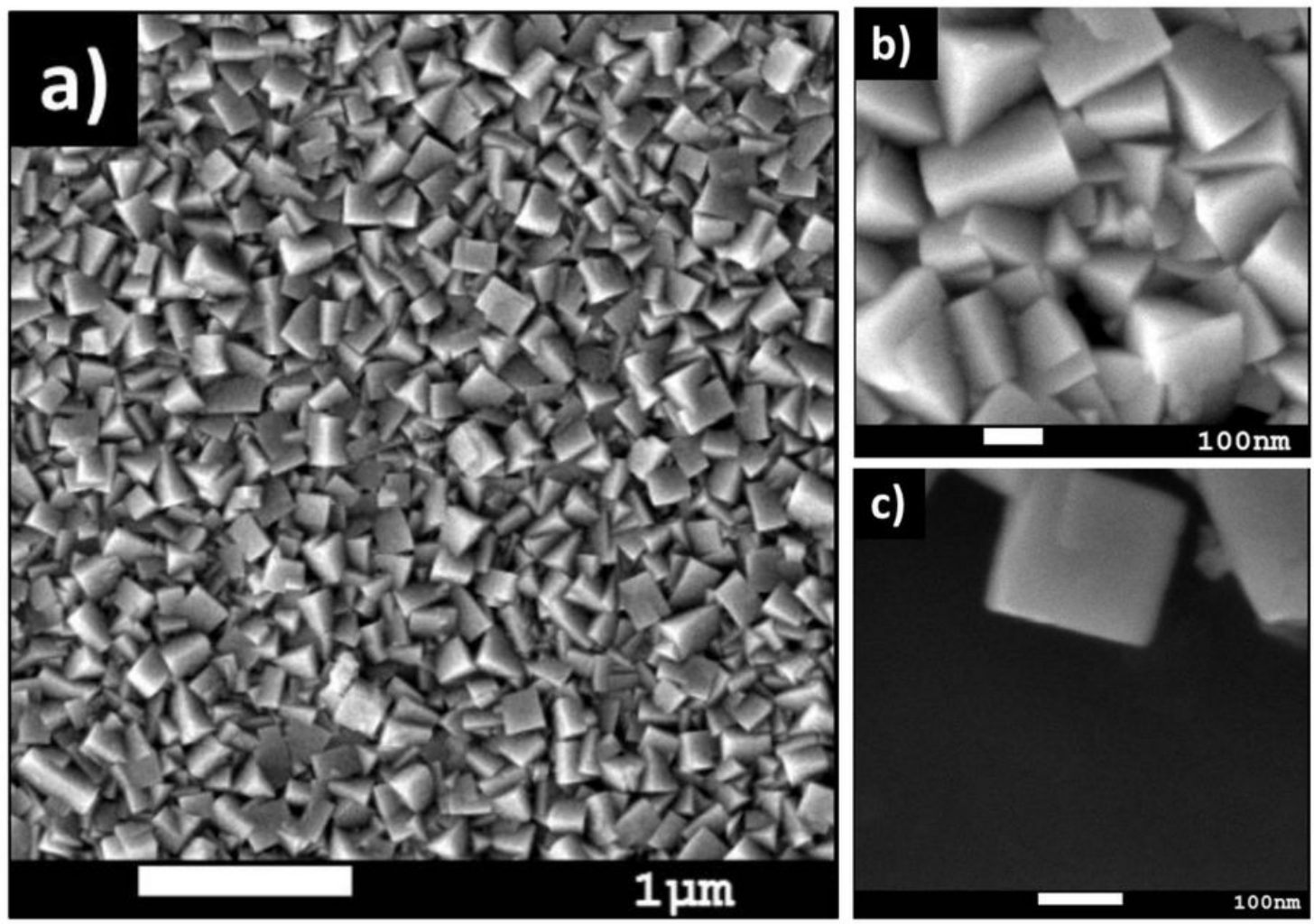

Figure 5

Top view of surface for PbS thin film to different magnifications, a) 10,000x, b) 50,000x and c) 100,000x. 


\section{Figure 6}

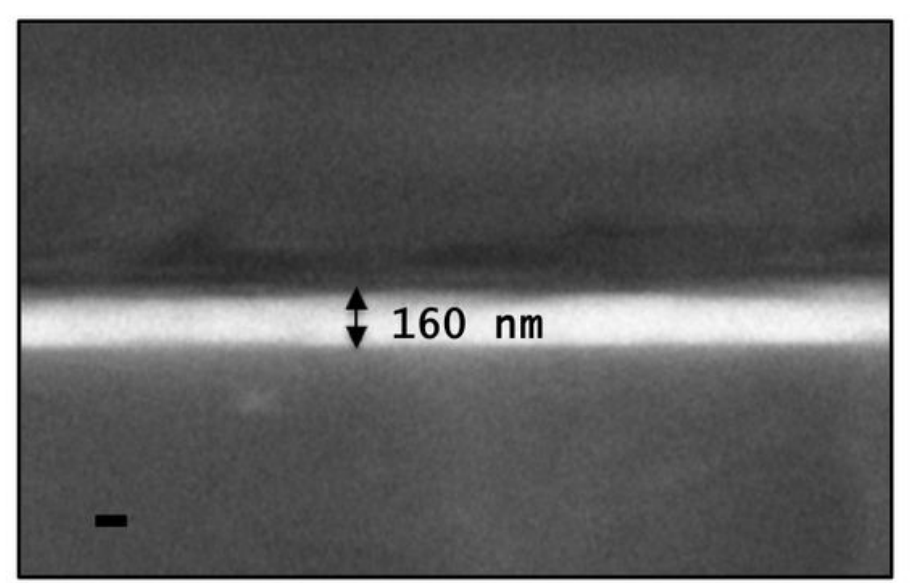

Figure 6

SEM image of cross-section views, estimating the thickness of PbS thin film. 


\section{Figure 7}
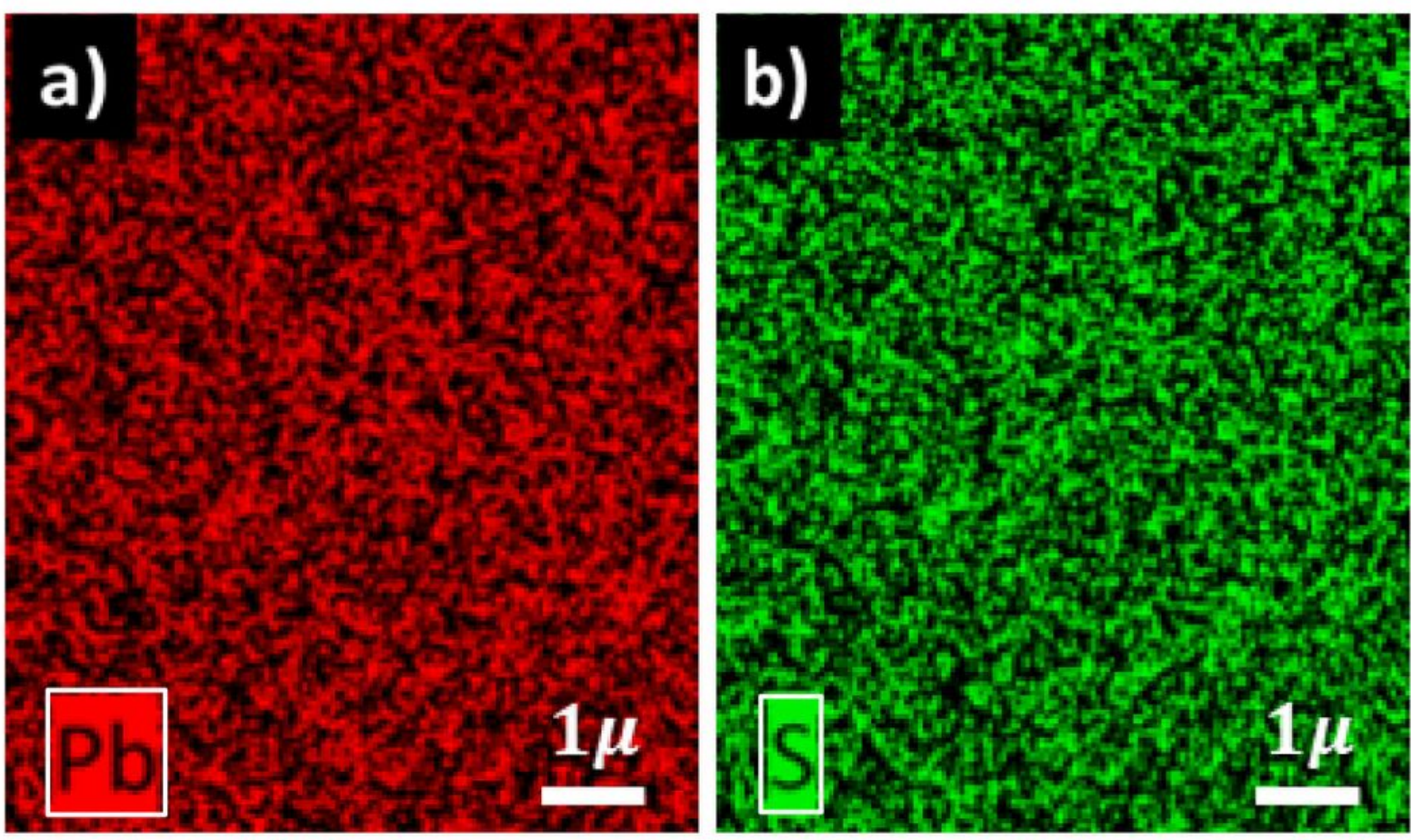

\section{Figure 7}

Elemental distribution on PbS thin film, a) lead and b) sulfide. 


\section{Figure 8}
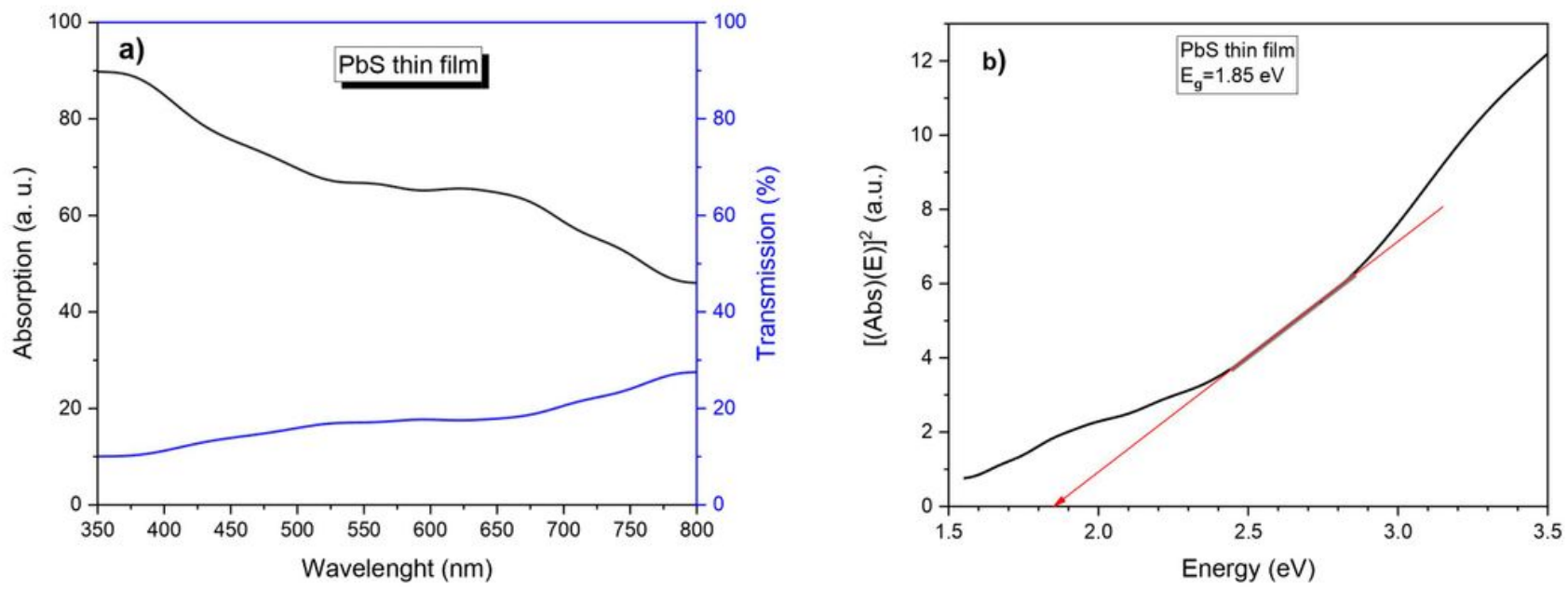

\section{Figure 8}

Part a) comparative complementary behaviors of absorption and transmission for a PbS thin film, and part b) graphical support for direct bandgap calculation. 


\section{Figure 9}

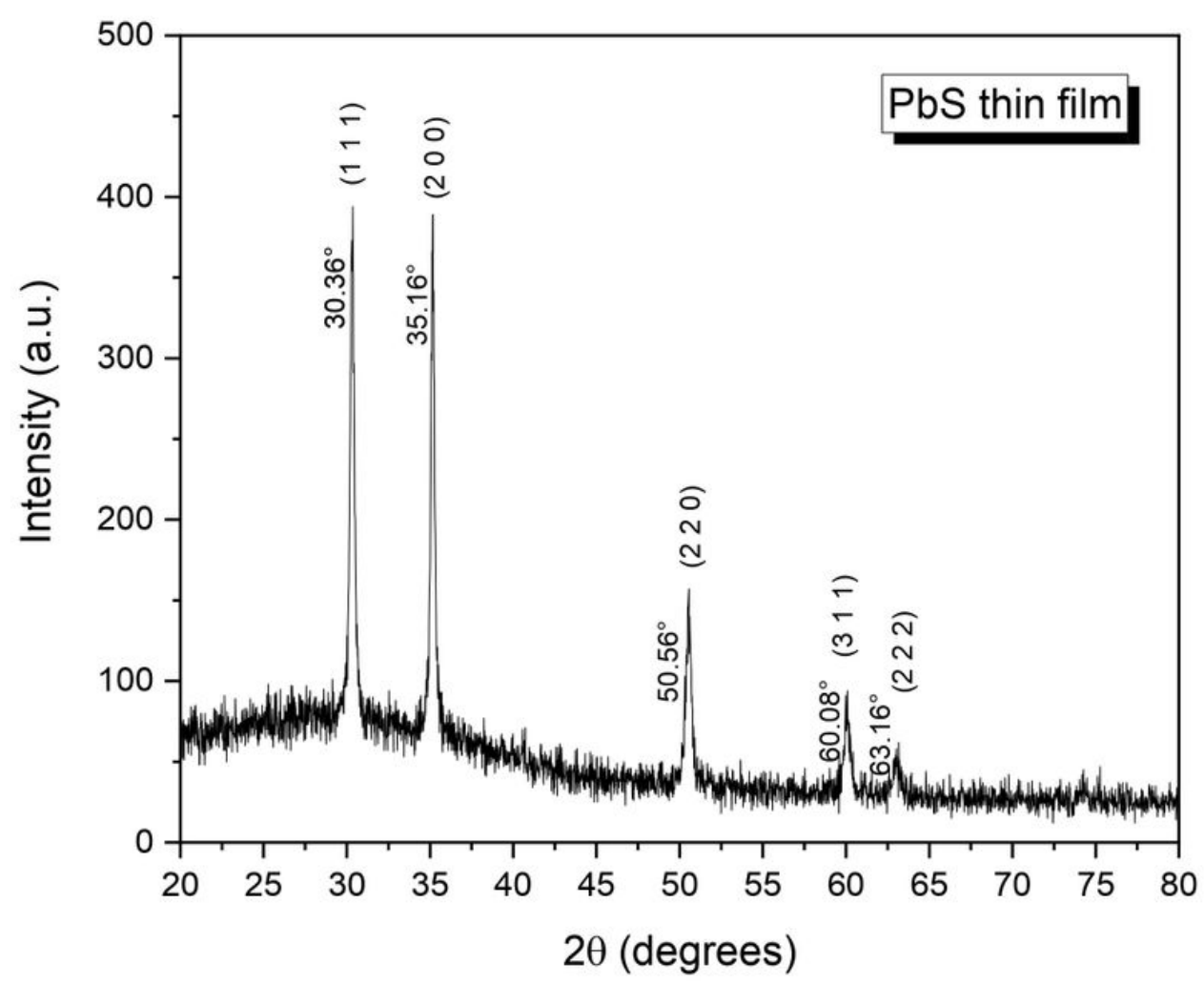

Figure 9

XRD pattern of polycrystalline PbS thin film deposited on a soda-lime glass substrate. 


\section{Figure 10}

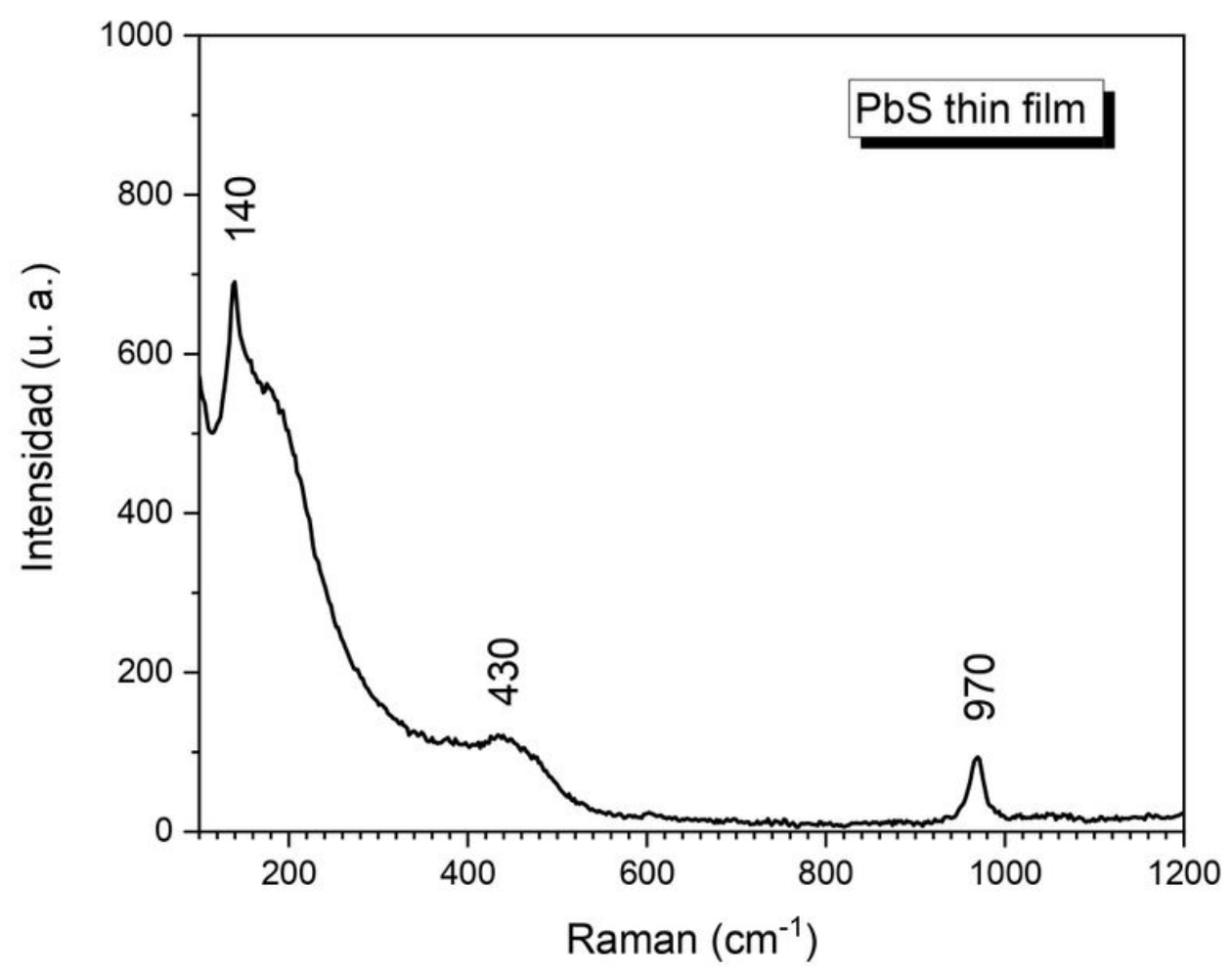

Figure 10

Raman dispersion spectroscopy was collected from synthesized PbS thin film, using a $488 \mathrm{~nm}$ laser, with three characteristic values corresponding to the PbS compound. 